\title{
Determinants of undernutrition among children aged 6 to 59 months in rural Zambézia Province, Mozambique: results of two population-based serial cross-sectional \\ surveys
}

Elizabeth S. Rose ${ }^{1}$, Meridith Blevins ${ }^{1,2,3}$, Lazaro González-Calvo ${ }^{1,2}$, Elisée Ndatimana², Ann F. Green ${ }^{1,2}$,

Melanie Lopez ${ }^{4}$, Omo Olupona ${ }^{5}$, Sten H. Vermund ${ }^{1,2,6}$, Troy D. Moon ${ }^{1,2,6^{*}}$ and For the Ogumaniha-SCIP Zambézia

Consortium

\begin{abstract}
Background: While many countries are transitioning from epidemics of undernutrition to overnutrition, Mozambique's very high $44 \%$ prevalence of stunting in children under age 5 years is cause for serious concern.

Methods: We conducted two population-based cross-sectional surveys of 4000 female heads of households each in Zambézia Province, Mozambique from August-September 2010 (Baseline) and April-May 2014 (Endline) as part of the USAID funded Strengthening Communities through Integrated Programs (SCIP) grant. Anthropometric measurements were collected on 560 children aged 6-59 months at Baseline and 912 children at Endline and classified as: "stunted," a height-for-age z-score less than -2; "wasted," weight-for-height z-score less than -2; and "underweight," weight-for-age $z$-score less than -2. Descriptive statistics and logistic regression using Stata 13.1 were used to examine factors associated with undernutrition.
\end{abstract}

Results: Of children under age 5 years, $43 \%$ were undernourished in 2010 and $55 \%$ in 2014. The most common form of undernutrition was stunting (39\% in 2010, $51 \%$ in 2014), followed by underweight (13\% in both 2010 and 2014), and wasting (7 \% in 2010, $5 \%$ in 2014). Child's age was found to have a non-linear association with stunting. Vitamin A supplementation was associated with a $31 \%(p=0.04)$ decreased odds of stunting. Children who were exclusively breastfed for at least 6 months had an $80 \%(p=0.02)$ lower odds of wasting in 2014 and $57 \%(p=0.05)$ decreased odds of being underweight in 2014. Introducing other foods after age 6 months was associated with a five-fold increased odds of wasting in 2014 ( $p=0.02)$; household food insecurity was associated with wasting (OR=2.08; $p=0.03)$ and underweight in $2010(\mathrm{OR}=2.31 ; p=0.05)$. Children whose mother washed her hands with a cleaning agent had a $40 \%(p=0.05)$ decreased odds of being underweight. Surprisingly, per point increase in household dietary diversity score, children had $12 \%$ greater odds of being stunted in $2010(p=0.01)$ but $9 \%$ decreased odds of being underweight in 2014 ( $p=0.02)$.

(Continued on next page)

\footnotetext{
* Correspondence: troy.moon@vanderbilt.edu

${ }^{1}$ Vanderbilt Institute for Global Health, Nashville, 2525 West End Avenue,

Suite 750, Nashville, Tennessee 37203, USA

${ }^{2}$ Friends in Global Health, Maputo, Mozambique

Full list of author information is available at the end of the article
} 
(Continued from previous page)

Conclusions: A combination of household and individual level factors was associated with undernutrition. As such, employment of multidimensional interventions should be considered to decrease undernutrition in children under 5 years old.

Keywords: Undernutrition, Stunting, Wasting, Mozambique, Rural, Children under 5 years of age, Trends

\section{Background}

Recent increases in globalization, urbanization, and the availability of processed foods high in sugars, sodium, and fat have contributed to a shift in focus from undernutrition to overnutrition in developing countries [1-3]. Despite this shift, undernutrition continues to be a major problem, particularly in rural Africa. Among lowand middle-income countries (LMICs), the prevalence of chronic malnutrition, or stunting (height-for-age z-score less than -2) was $28 \%$ in 2011 [4]. In the same year, countries in sub-Saharan African reported a prevalence of stunting of $40 \%$ and in Mozambique in particular, the prevalence was even higher at $44 \%$ [5-7]. Mozambique ranks 178 out of 187 countries on the United Nations Development Program (UNDP) 2014 Human Development Index [8] and its prevalence of stunting is one of the highest in Africa [9]. In terms of the total number of children who are stunted, in 2011 Mozambique ranked $16^{\text {th }}$ in the world and $10^{\text {th }}$ in Africa, with an estimated 1.65 million stunted children, [10] though the country ranks $49^{\text {th }}$ in population [11]. The determinants of undernutrition, which range from distal-level socio-economic and political factors to proximal-level food practices, disease frequency, and health care, have been studied in various locations worldwide, [4, 12-16] but few studies [5, 17] include Mozambique.

As the period for reaching the Millennium Development Goals (MDGs) draws to a close in 2015, assessing the reasons behind why goals were or were not reached can guide future human development efforts in LMICs. Insufficient nutrition is a cross-cutting condition that both directly and indirectly impedes progress towards MDGs one (eradicating extreme poverty and hunger), two (achieving universal education), and four (reducing child mortality) [18]. In 2012, an estimated 162 million (25\%) children under five suffered from stunting worldwide, with an additional 112 million (15\%) children classified as underweight (weight-for-age z-score less than -2) [19].

Maternal prenatal nutrition and poor intake of micronutrients, such as vitamin A, by the infant in the first 1000 days of life can cause irreversible developmental damage and impediments in physical and cognitive growth that last into adulthood [9, 20]. Poor nutrition has been correlated with lower cognitive performance in school and decreased success in the labor market as an adult, which further perpetuates the cycle of poverty and undernutrition [18]. Undernutrition, which includes stunting, underweight, and wasting (weight-for-height $\mathrm{z}$-score less than -2), is the largest preventable cause of death among children under five [20] and is directly or indirectly attributed to 3.1 million (45 \%) deaths in this population [4]. Ninety-eight percent of undernourished children live in developing countries and while the prevalence of undernutrition has decreased across almost all world regions over the past two decades, rates have increased in Africa, mandating a better understanding of the determinants of undernutrition in this geographical setting [19].

Compounding the poor nutritional indices of Mozambique described above are the salient disparities in health care, outcomes, and budgeting allocations among the country's provinces. The centrally-located Zambézia Province (Fig. 1) has the lowest per capita budget for health and education, the lowest access to safe water (26\% coverage, 2009), the highest sanitation deprivation among children (73\%, 2008), the highest poverty headcount $(71 \%, 2008 / 9)$, and the highest deprivation-based child poverty rate $(64 \%, 2008)$ [9]. This province also has some of the lowest performance indicators for health outcomes in the country, including the highest under 5 mortality rate (206 per 1000 live births, 10 year average 1998-2008) and among the highest child stunting rates (46\%, 2008) [9]. Zambézia Province has been labeled as a development "priority province" and as such, numerous national and international programs have been undertaken, yet undernutrition rates have remained relatively stable [9]. Aligning with the World Health Organization (WHO) recommendations that countries place the management of undernutrition as a public health priority, we sought to study the determinants of undernutrition among children under 5 years of age, over a four-year period, so as to inform future interventions and health practices that aim to reduce the prevalence of undernutrition in Zambézia Province [21].

\section{Methods}

The Ogumaniha Project was a 5-year program that began implementation in Zambézia Province in late 2009. Ogumaniha, which means "united for a common purpose" in the local language Echuabo, broadly aimed 


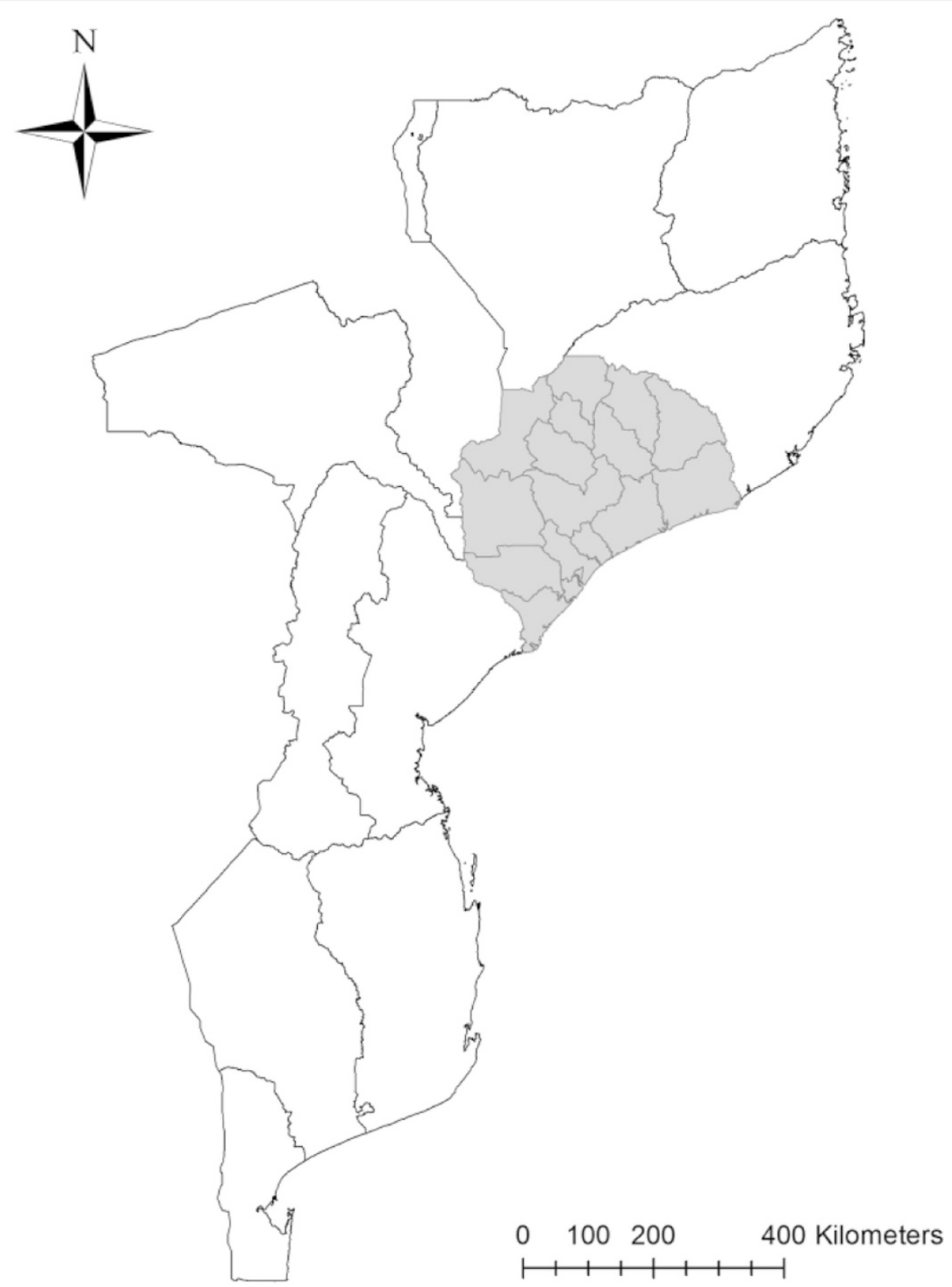

Fig. 1 Map of Mozambique with Zambézia Province (shaded) and its districts. Charlotte Buehler; May 27, 2015; Vanderbilt Institute for Global Health; Projection: WGS 1984 Web Mercator Auxiliary Sphere. Permission has been granted by the copy-right holder for publication of this figure in an open access journal

to reduce poverty and improve the health and livelihoods of people living in Zambézia Province through integrated and sustainable community-based programming [22]. Households within each enumeration area (EA) received a variety of multidimensional interventions over a 5-year period. The Project was funded under USAID's Strengthening Communities through Integrated Programming (SCIP) grant and was implemented through a consortium of organizations led by World Vision from 2009 to 2014. This study is an extension of the monitoring and evaluation component of the Ogumaniha Project and is an assessment of crosssectional survey data collected at Baseline (August and September 2010) and Endline (April and May 2014). At both Baseline and Endline the same questionnaire was utilized. While we did not collect survey responses from the same households in both surveys, we utilized the same sampling methodology and returned to the same EAs as in Baseline. The two-stage cluster sampling design employed in this study made use of the 
Government of Mozambique's sampling frame that was created for all national surveys and is based on 2007 census results. Further details about the sampling methodology, electronic data collection using mobile phones, Open Data Kit, and management protocols have been published elsewhere [22]. In brief, the Ogumaniha survey tool collects information on over 500 variables in 8 dimensions and was developed by a team of multidisciplinary researchers. The survey was designed to collect information from the female head of household. Mobile survey teams conducted interviews in 259 enumeration areas (EA) across 14 of Zambézia's 17 districts. EA selection was stratified by district with probability proportional to size. The entire sample size is representative of the province, while three districts were over sampled for improved precision and decreased survey costs. Fourteen teams with a team leader and four interviewers collected the data. Data were collected using a mobile cell phone. Interviewers received intensive training on the use of mobile phones for data collection prior to survey implementation.

In households with at least one child 0-12 months and/or one child 13-59 months, one child was randomly selected from each age group and weight and length measurements were collected following the WHO recommendation for children's nutritional anthropometry [22]. Undernourished children were then classified by the following anthropometric groupings using standardized measurements developed by the WHO in 2006 as reference data: stunted, a height-for-age $\mathrm{z}$-score less than -2; wasted, a weight-for-height $\mathrm{z}$-score less than -2 ; and underweight, a weight-for-age $\mathrm{z}$-score less than -2 .

Covariates were selected a priori based on an extensive literature review and UNICEF's malnutrition conceptual framework [23]. Selected variables were child's age, breastfeeding practices, vitamin A supplementation, household income, food insecurity, household dietary diversity score (HDDS), water and sanitation, and child having diarrheal illness. These potential variables of undernutrition were self-reported through a questionnaire conducted with the female head of household. Vitamin A supplementation was determined by reviewing the child's immunization card, when available. Breastfeeding practice covariates included length of exclusive breastfeeding and child's age when supplemental feeding began, both reported in months. Family income was reported in meticais $(1 \mathrm{USD} \approx 36 \mathrm{MZN}$ in August 2010 and $1 \mathrm{USD} \approx 31 \mathrm{MZN}$ in April 2014) and food insecurity was defined as having had no food at all to eat at least once within the month prior to survey. Household dietary diversity was determined by summing the number of unique food categories consumed by household members over a 7 day recall period, as outlined by the Food and Nutrition Technical Assistance Project (FANTA) [24].
Water and sanitation factors were determined by whether the household used a river as a drinking water source, which was considered an unsafe water source in this study, and whether the female head of household washed her hands with soap, detergent, or ashes on the day prior to interview. Child having a diarrheal illness in the past 30 days was self-reported by the interviewee.

Descriptive statistics were calculated for continuous variables as weighted median with interquartile range and for categorical variables as weighted percentages, with each observation being weighted by the inverse of the child sampling probability. Multivariable logistic regression models with robust covariance to account for clustering in the sample design were used to estimate risk factors for malnutrition outcomes. Covariates included all aforementioned causes of undernutrition: vitamin A supplementation, child age, breastfeeding practices, household income, household dietary diversity, household food security, water and sanitation, and child history of diarrheal illness. Restricted cubic splines were used to relax linearity assumptions for all continuous covariates (e.g., child age and household income). Where there was little evidence of non-linearity, assessed using likelihood-ratio tests, covariates were retained as linear exposures in the model [25]. The significance level for all testing was two-sided and set at 0.05. Stata 13.1 was used for all data analyses described above [26].

The study was approved by the Inter-institutional Bioethics Committee for Health of Zambézia Province (CIBS-Zambézia), Mozambique and the Vanderbilt University Institutional Review Board (IRB). Informed verbal consent was obtained from the female head of house before the interview and child measurements were conducted.

\section{Results}

A total of 560 children aged 6-59 months were measured at baseline in 2010 and 912 at Endline in 2014. Prevalence of undernutrition was $43 \%$ in 2010 compared to $55 \%$ in 2014 (Tables 1 and 2). The most common form of undernutrition was stunting (39\% in 2010, $51 \%$ in 2014), followed by underweight (13\% in both 2010 and 2014), and wasting (7\% in 2010, $5 \%$ in 2014). In 2010, no difference in age (median 36 months) was seen between children classified as undernourished compared to those not undernourished, while in 2014 the median age was 24 months for undernourished children compared to 18 months for those not undernourished $(p=0.01)$. At both time points, $80 \%$ of children measured came from rural communities. Likewise more than $80 \%$ of all children were from households that reported being of some form of Christian religion (Catholic, Evangelical/Pentecostal, or Protestant). Additionally at both times points, approximately $90 \%$ reported a 
Table 1 Descriptive statistics of children 6-59 months, 2010 survey, Zambézia, Mozambique

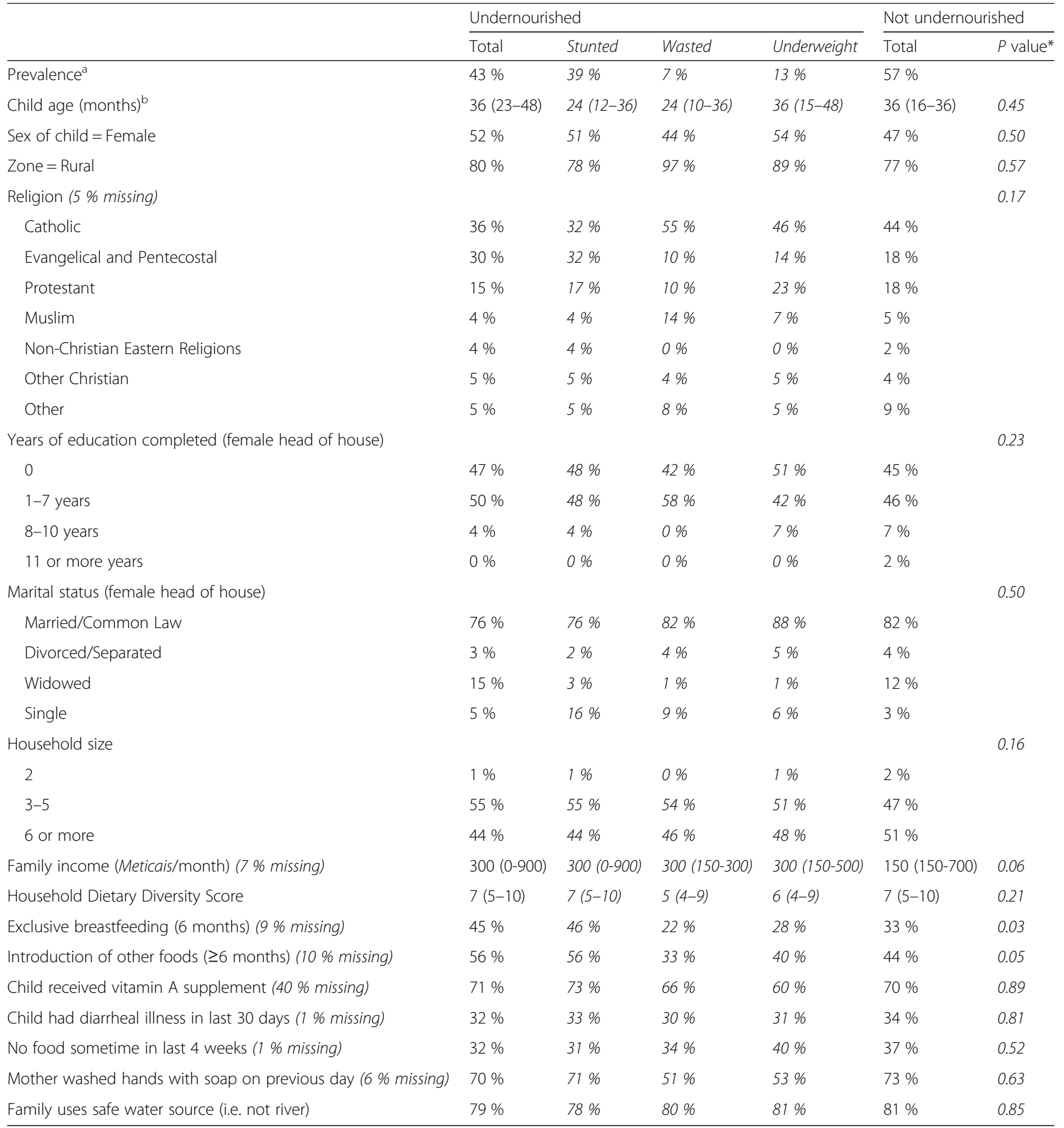

* $P$ value refers to the Pearson Correlation coefficient between not undernourished and undernourished

${ }^{a}$ Categorical variables are reported as weighted percentages, with each observation being weighted by the inverse of the child sampling probability. Due to rounding, some categories may not total $100 \%$

${ }^{b}$ Continuous variables are reported as weighted estimates of median (interquartile range), with each observation being weighted by the inverse of the child sampling probability

maternal education of 7 years or less (Tables 1 and 2). Median length of exclusive breastfeeding in months increased from 4 months (IQR 3-6) in 2010 to 6 months (IQR 4-6) in 2014 (data not shown).
In multivariable models of analysis, the characteristics most significantly associated with being stunted in 2010 were higher household income (per 100 MZN per month increase) and higher household dietary 
Table 2 Descriptive statistics of children 6-59 months, 2014 survey, Zambézia, Mozambique

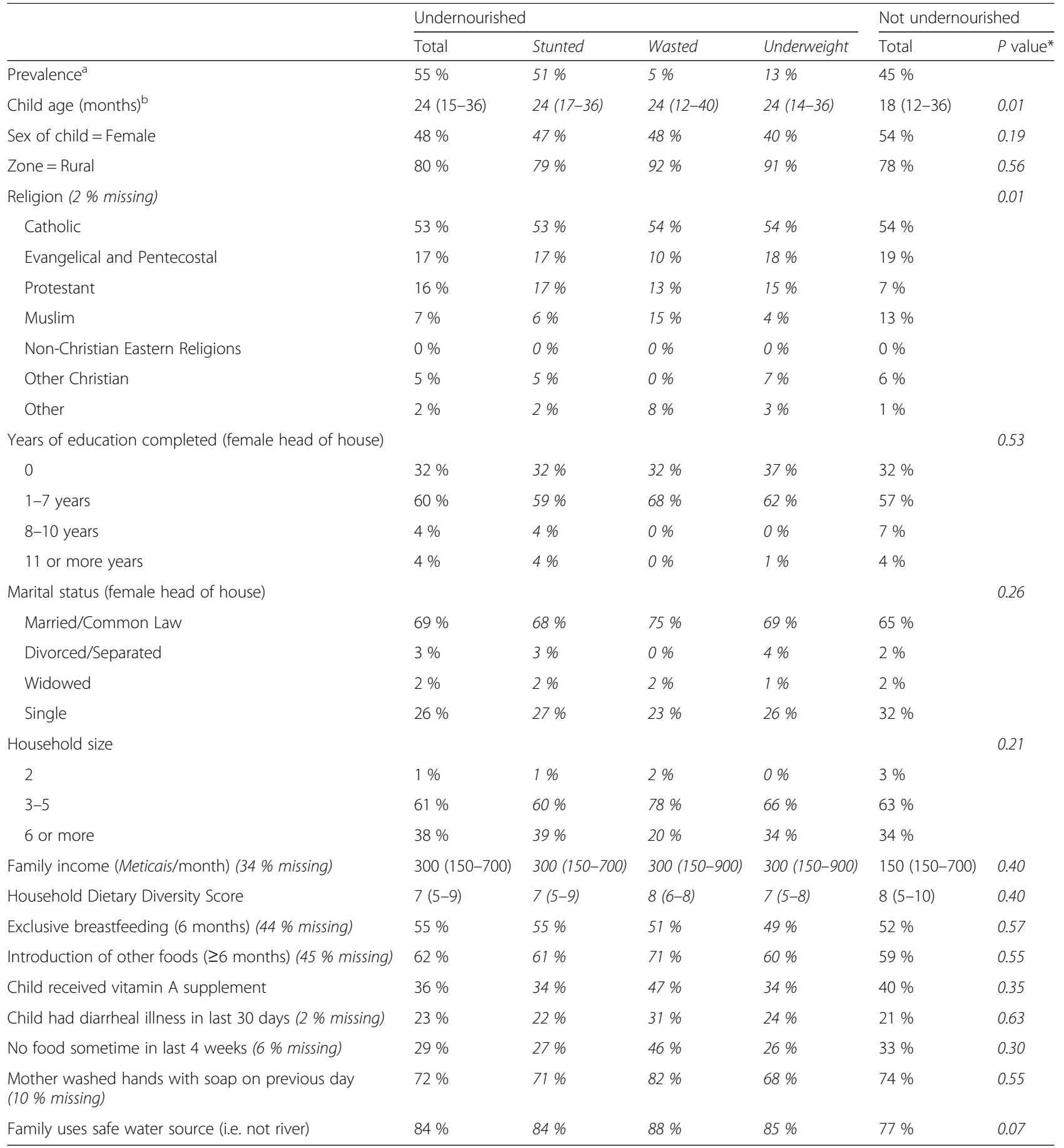

* $P$ value refers to the Pearson Correlation coefficient between not undernourished and undernourished

${ }^{a}$ Categorical variables are reported as weighted percentages, with each observation being weighted by the inverse of the child sampling probability. Due to rounding, some categories may not total $100 \%$

${ }^{b}$ Continuous variables are reported as weighted estimates of median (interquartile range), with each observation being weighted by the inverse of the child sampling probability

diversity score (HDDS) (Table 3). By contrast, in 2014, age was the only characteristic that had a statistically significant association with stunting. Compared to children who were 12 months old, children who were 24 months old had a $94 \%$ increased odds of being stunted $(\mathrm{OR}=1.94 ; 95 \% \mathrm{CI}=1.50-2.51)$, those who were 36 months had a $134 \%$ increased odds $(\mathrm{OR}=2.34 ; 95 \% \mathrm{CI}=1.63-3.37)$, and children 48 months 
Table 3 Logistic regression of stunted status (height-for-age z-score <-2), Zambézia Province, Mozambique

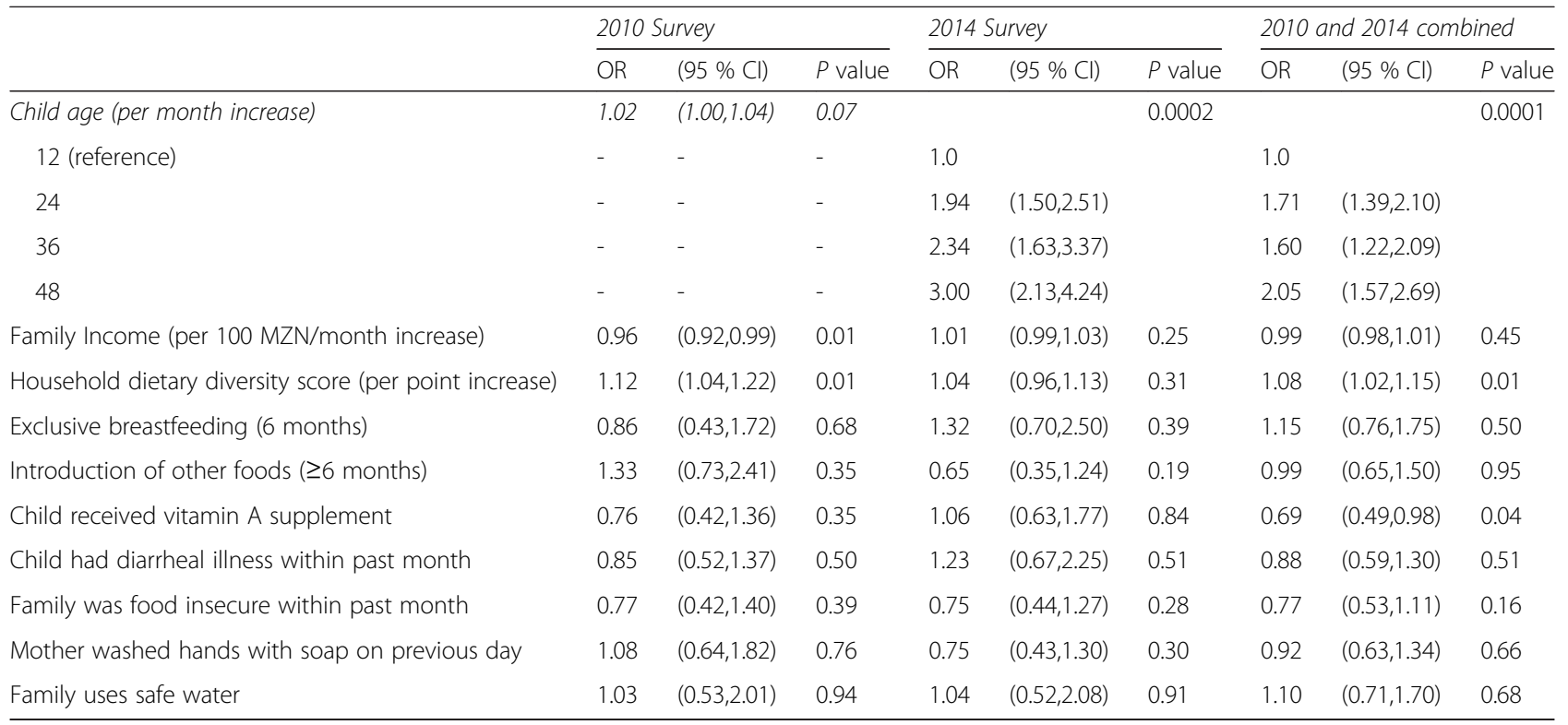

old had three-fold the odds of being stunted $(\mathrm{OR}=3.00$; $95 \% \mathrm{CI}=2.13-4.24) \quad(p=0.0002)$. When 2010 and 2014 data were aggregated in a multivariable model of analysis, age (non-linear association, Fig. 2) and increasing HDDS maintained a significant association with stunting. Additionally, vitamin A supplementation was found to be protective against stunting, with children who received vitamin A supplementation having a $31 \%$ decreased odds of being stunted $(\mathrm{OR}=0.69 ; 95 \% \mathrm{CI}=0.49-0.98$; $p=0.04$ ).

In 2010, none of the variables tested in multivariable models had a statistically significant association with wasting. However, a child's mother washing her hands with soap on the day prior to measurement trended towards being protective $(\mathrm{OR}=0.43 ; 95 \% \mathrm{CI}=0.18-1.05$; $p=0.06$ ) (Table 4). In 2014, children who exclusively breastfed for at least 6 months had an $80 \%$ decreased odds of being wasted $(\mathrm{OR}=0.20 ; 95 \% \mathrm{CI}=0.05-0.74$; $p=0.02$ ). Moreover, children measured in 2014 who were introduced to supplemental foods after 6 months of age had over a five-fold increase in the odds of being wasted $(\mathrm{OR}=5.37 ; 95 \% \mathrm{CI}=1.28-22.55 ; p=0.02)$. Additionally, in 2014 children from households that faced food insecurity had over three-fold increased odds of being wasted ( $\mathrm{OR}=3.70 ; 95 \% \mathrm{CI}=0.90-15.10$; $p=0.07$ ), though it was not statistically significant.

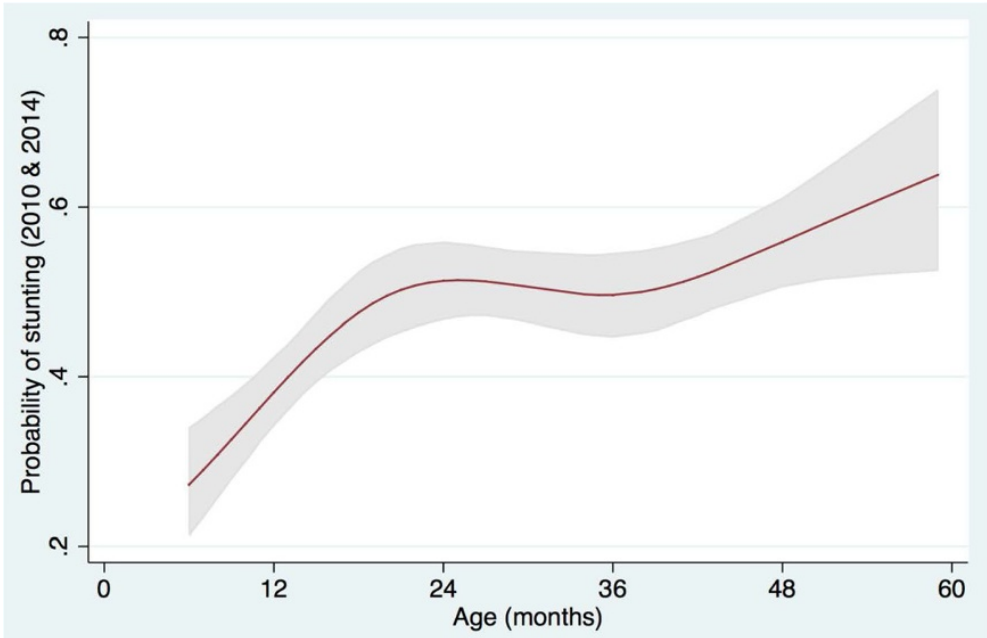

Fig. 2 Restricted cubic spline of aggregated 2010 and 2014 data revealing the adjusted association between child's age (months) and the probability of stunting among children 6-59 months. The $95 \%$ confidence interval is represented by the gray area 
Table 4 Logistic regression of wasted status (weight-for-height z-score <-2), Zambézia Province, Mozambique

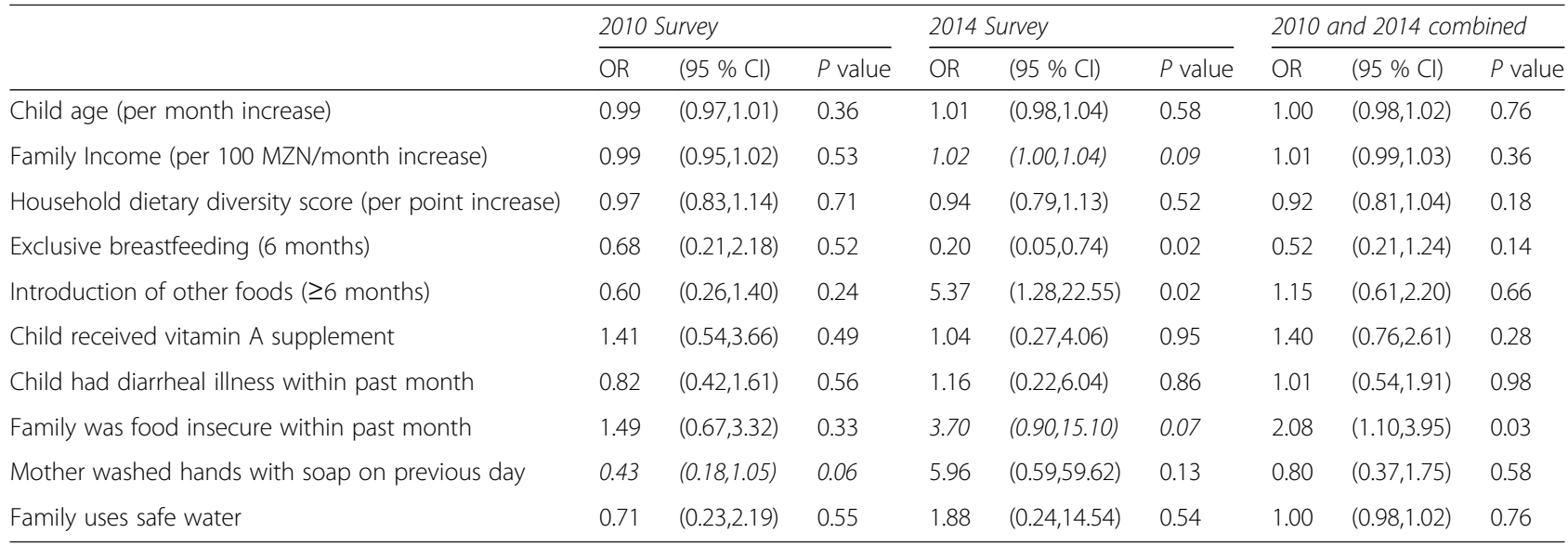

When 2010 and 2014 data were aggregated, children from households that were food insecure had twice the odds of being wasted $(\mathrm{OR}=2.08 ; 95 \% \mathrm{CI}=1.10-3.95$; $p=0.03$ ).

In 2010, household food insecurity within the past month resulted in over twice the odds of being underweight in multivariable analysis $(\mathrm{OR}=2.31 ; 95 \%$ $\mathrm{CI}=1.02-5.23 ; p=0.05)$ and a child's mother washing her hands with soap in the day prior to interview trended towards being protective against underweight, though did not reach statistical significance $(\mathrm{OR}=0.44$; $95 \% \mathrm{CI}=0.19-1.06 ; p=0.07$ ) (Table 5). In 2014, both increasing HDDS as well as exclusive breastfeeding for at least 6 months had protective associations against being underweight, with the latter reducing odds by $57 \%(\mathrm{OR}=0.43 ; 95 \% \mathrm{CI}=0.18-1.02 ; p=0.05)$. When 2010 and 2014 data were analyzed in aggregate, increasing age was significantly associated with being underweight, while exclusive breastfeeding for at least 6 months and the child's mother washing her hands with soap on the day prior to interview showed a protective association against being underweight, decreasing odds by $47 \%$ and $40 \%$, respectively $(\mathrm{OR}=0.53 ; 95 \% \mathrm{CI}=0.29$ $0.97 ; p=0.04$ and $\mathrm{OR}=0.60 ; 95 \% \mathrm{CI}=0.36-1.01 ; p=0.05$, respectively).

\section{Discussion}

The prevalence of stunting in our study was "very high" as per WHO [27] classification and also in comparison to worldwide rates of stunting in other LMICs that range from 5 to $65 \%$ [28]. Recorded rates of wasting and underweight, as defined by the WHO, were categorized as "poor" and "medium prevalence," respectively [27]. Stunting prevalence is of particular concern since it reflects long-term structural factors of undernutrition and can serve as an indicator of a population's wellbeing [9]. The WHO [28] and other studies [9, 23] have found that stunting prevalence increases with age from birth until 24 to 36 months and then plateaus. In contrast, our data found that children with increasing age,

Table 5 Logistic regression of underweight status (weight-for-age z-score <-2), Zambézia Province, Mozambique

\begin{tabular}{|c|c|c|c|c|c|c|c|c|c|}
\hline & \multicolumn{3}{|c|}{2010 Survey } & \multicolumn{3}{|c|}{2014 Survey } & \multicolumn{3}{|c|}{2010 and 2014 combined } \\
\hline & $\overline{\mathrm{OR}}$ & $(95 \% \mathrm{Cl})$ & $P$ value & $\overline{\mathrm{OR}}$ & $(95 \% \mathrm{Cl})$ & $P$ value & $\overline{\mathrm{OR}}$ & $(95 \% \mathrm{Cl})$ & $P$ value \\
\hline Child age (per month increase) & 1.01 & $(0.99,1.03)$ & 0.34 & 1.03 & $(1.01,1.05)$ & 0.01 & 1.02 & $(1.00,1.04)$ & 0.02 \\
\hline Family Income (per 100 MZN/month increase) & 1.01 & $(0.97,1.04)$ & 0.71 & 1.01 & $(0.99,1.03)$ & 0.41 & 1.01 & $(0.99,1.02)$ & 0.39 \\
\hline Household dietary diversity score (per point increase) & 1.06 & $(0.92,1.23)$ & 0.39 & 0.91 & $(0.83,0.99)$ & 0.02 & 0.96 & $(0.89,1.04)$ & 0.37 \\
\hline Exclusive breastfeeding (6 months) & 0.43 & $(0.14,1.28)$ & 0.13 & 0.43 & $(0.18,1.02)$ & 0.05 & 0.53 & $(0.29,0.97)$ & 0.04 \\
\hline Introduction of other foods ( $\geq 6$ months) & 0.57 & $(0.25,1.32)$ & 0.19 & 1.80 & $(0.75,4.36)$ & 0.19 & 1.01 & $(0.56,1.83)$ & 0.97 \\
\hline Child received vitamin A supplement & 1.17 & $(0.55,2.51)$ & 0.68 & 1.03 & $(0.52,2.03)$ & 0.93 & 0.92 & $(0.60,1.40)$ & 0.70 \\
\hline Child had diarrheal illness within past month & 0.63 & $(0.32,1.25)$ & 0.19 & 1.07 & $(0.45,2.55)$ & 0.88 & 0.83 & $(0.49,1.43)$ & 0.51 \\
\hline Family was food insecure within past month & 2.31 & $(1.02,5.23)$ & 0.05 & 0.90 & $(0.40,2.01)$ & 0.80 & 1.47 & $(0.87,2.49)$ & 0.15 \\
\hline Mother washed hands with soap on previous day & 0.44 & $(0.19,1.06)$ & 0.07 & 0.65 & $(0.30,1.39)$ & 0.27 & 0.60 & $(0.36,1.01)$ & 0.05 \\
\hline Family uses safe water & 0.82 & $(0.32,2.09)$ & 0.68 & 1.58 & $(0.60,4.21)$ & 0.36 & 1.12 & $(0.58,2.18)$ & 0.74 \\
\hline
\end{tabular}


above 2 years, were at highest risk of being stunted and/ or underweight. The aggregated 2010 and 2014 stunting data reveals a non-linear association between stunting and child's age (Fig. 2). This phenomenon is likely due to a variety of factors. First, Ogumaniha interventions targeted poor nutrition through promotion of exclusive breastfeeding for children 6 months and younger, training of community volunteers on the preparation of foods high in essential micronutrients, and identification of children with moderate undernutrition within communities for caloric supplementation and/or referral to a health facility for treatment if necessary. The lower odds of stunting in younger children may be evidence that they received and positively responded to these interventions, while the higher odds of stunting at later ages (36 to 59 months) may largely be due to these children having past the window of opportunity to prevent the permanent effects of undernutrition when interventions were introduced. Second, as a child is weaned from the breast, their growth and nutrition may be negatively affected by the mother's inability to continue meeting the nutritional needs of the growing child with breast milk alone and/or by either the introduction of high-starch, low-protein foods or by a delayed introduction of supplemental foods. The median length of time for exclusive breastfeeding in our study increased between 2010 and 2014, thus potentially delaying the negative chronic effects of undernutrition as children were weaned later. This is further supported by our finding of an $80 \%$ decreased odds of wasting in 2014 in children that exclusively breastfed for a minimum of 6 months and a five-fold increased odds of wasting in children who had delayed introduction of supplemental food.

Our finding in multivariable analysis of a protective association between receipt of vitamin A supplementation and decreased odds of stunting is consistent with similar studies in LMICs [4, 20, 23, 29-31]. These studies found that micronutrient deficiencies, including vitamin $\mathrm{A}$, iron, and zinc, had a significant impact on a child's height potential. Our survey questionnaire did not ask about zinc and iron supplementation, as they are not commonly administered in the province. In 2010, $70 \%$ of all children reported receipt of vitamin A supplementation while only $37 \%$ reported receiving supplementation in 2014. Although this stark difference could be attributed to missing data (40\% was missing in 2010), it is vital to increase the coverage of vitamin A supplementation or consider the implications of introducing foods fortified with vitamin A, such as sweet potatoes [32].

Higher proportions of children with undernutrition came from households with a higher monthly income, though no associations were detected between income and either stunting or wasting in multivariable analysis. This counterintuitive finding may result from the fact that there is likely no meaningful difference between the measured 300 versus 150 meticais per month (a difference of approximately \$4 USD) in terms of purchasing power at a household level. Additionally, almost one quarter of interviewees did not know their household income, which is common among subsistence farmers and those who rely on seasonal and informal markets. In this setting, non-monetary measures of poverty, such as the multidimensional poverty index which classifies one's poverty based on measures such as health, education, and material means may be more appropriate [33].

The perceived association between increased stunting and greater household dietary diversity could be explained by an inability to capture the individual child's dietary diversity with the survey tool used. Anecdotal evidence from Zambézia Province suggests there is a hierarchical determination of diet, such that male adults within a given household may have a more diverse diet than females and children respectively, and that natural children within a household may have better diets than children taken into the home as orphans or from extended family, despite these foods being available in the household. While providing a potential measure of overall household access to resources, dietary diversity may not be a sensitive marker of under-five malnutrition, particularly in infants younger than 9 months, who may not eat all foods available in the household or who are exclusively breastfeeding.

Multivariable analysis revealed that being food insecure more than doubled the odds of being underweight in 2010 and wasted when analyzed in aggregate. This finding aligns with our expectations because wasting and underweight are traditionally used to monitor shortterm deprivation of food, while stunting reveals reoccurring nutrient inadequacies over a lengthier period of time [23, 34, 35].

Hand washing practices that included the using of a cleaning agent decreased the odds of being underweight by $40 \%$. Although $95 \%$ of interviewees reported washing hands the previous day, only $72 \%$ washed their hands with soap, detergent, or ashes. The use of a cleaning agent is necessary to remove grime and bacteria from the hands, and ashes have been reported to be an effective, low-cost alternative to soap in preventing the spread of diarrheal illness [36]. Future interventions in Zambézia Province should focus on education and economic interventions that will continue to increase the use of cleaning agents.

In our multivariable analysis, there was little evidence that the use of safe water was associated with undernutrition. Eighty percent of households used safe water and this lack of variability in the data may have made it difficult to observe a correlation between this variable and the outcome variables. Despite using safe water, roughly 
$30 \%$ of children were reported as having diarrheal illness within the past month, regardless of nutritional status. We did not detect a statistically significant association between diarrheal illness and undernutrition, although recurring infectious disease and unhealthy environmental conditions have been correlated with stunting in other settings $[4,16,23,30,37]$. This undetectable correlation in our study could be attributed to the aforementioned lack of variability in the population. Future directions of study should include collecting bio-specimens to determine if environmental enteropathy, a subclinical condition of the gut that has been found to be an intermediary pathway connecting poor hygiene to growth deficits [38] plays a role in the undernutrition of this population.

Strengths of this study include a large sample size and survey design that was representative of the province. Survey interviewers were Zambézia residents that administered the surveys in one of five local languages known to the interviewee. The models in the analyses were developed a priori and tested non-linearity of covariates with restricted cubic splines. A study limitation was that the breastfeeding questions referred to the female head of household's actions with her youngest child, which may not have been the child that was randomly selected for measurement. We assumed that practices were kept consistent among children, but there is a possibility of variance that was not accounted for in the data. Moreover, survey design was more heavily weighted towards questions that measured household level factors of undernutrition than for individual level factors. As such, it is likely we did not capture those individual level determinants that would prove important in impacting the high rates of undernutrition in this population, such as maternal undernutrition, birth weight, poor feeding practices, infectious disease specific history, and duration of disease. A final limitation of this study is the timing of the baseline and program-end surveys as they were conducted at two distinct periods of the year. Data collected in 2014 occurred at the end of the rainy, or "hunger," season, which may have influenced the increased prevalence of stunting as well as income and food related variables, such as dietary diversity and food insecurity. However, stunting is the result of long-term food deprivation, so it is likely that the timing of surveys had a low impact on this outcome variable.

\section{Conclusion}

Almost half of studied children aged 6-59 months in Zambézia Province were undernourished, revealing the need for sustained efforts to ameliorate this high prevalence rate. Of particular concern is the high rate of stunting that increased from 2010 to 2014. Intensified efforts to increase rates of vitamin A supplementation should be implemented, as well as other disease prevention measures such as interventions aimed at sustaining high rates of vaccine uptake. Interventions related to breastfeeding and hand washing practices as well as decreasing the extraordinary level of food insecurity that is prevalent throughout Zambézia Province should be implemented to help to reduce the prevalence of wasting and underweight. Future studies are needed to better explore local customs related to inter-household dietary diversity patterns, specifically focused on children under 5 years old. Ogumaniha's multidimensional interventions may have contributed to the decreased rate of poverty and food insecurity from 2010 to 2014 . Our study provided evidence that a combination of factors were associated with undernutrition. As such, employment of multidimensional interventions should be considered to decrease undernutrition in children under 5 years old.

\section{Competing interests \\ The authors declare that they have no competing interests.}

\section{Authors' contributions}

$E R$ and $M B$ participated in drafting and revising the manuscript, performing statistical analysis, and interpretation of findings. TM, MB, AG, EN, LG, ML, OO, and $\mathrm{SV}$ were involved in design of the study, interpretation of findings, drafting and revising the manuscript. $L G, T M, A G$, and $M B$ participated in data acquisition. All authors read and approved the final manuscript.

\section{Acknowledgments}

We acknowledge the members of the Ogumaniha monitoring and evaluation team including: Reginaldo Muluco, Iranett Manteiga, Ofelia Santiago, Faride Mussagy, Aurélio André Andate, Luciano Bene, Ezequiel Ossemane, Samuel Badaga, Oliveira Gada, Nelson Godinho, Arnete Alfredo, Mamudo Namuera, Helder Mundereia, Maximiana Paiva, Renato Lucia, Silvia Peixe, Quiteria Fernando, Fernando Cinco Reis, Berta Alice Fareto, Joaquina Vicente, Elias Impulula, Mussagy Massange, Zumira Abudu, Cremildo Mugas,Fatima Paúlo, Teofilo Jaime, Tánia de Morais, Elisée Ndatimana, Jeff Weiser, and Lara Vaz. We acknowledge the following collaborators of the Ogumaniha-SCIP Zambézia Consortium: Rodolfo Henriquez of World Vision-Mozambique, Luís Armazia of Adventist Development and Relief Agency-Mozambique, Hou Sei Carlos of International Relief and DevelopmentMozambique, Gloria Ekpo of World Vision-USA, and Ezequiel Barreto Ossemane of Friends in Global Health-Mozambique.

\section{Author details \\ ${ }^{1}$ Vanderbilt Institute for Global Health, Nashville, 2525 West End Avenue, Suite 750, Nashville, Tennessee 37203, USA. ${ }^{2}$ Friends in Global Health, Maputo, Mozambique. ${ }^{3}$ Department of Biostatistics, Vanderbilt University, Nashville, Tennessee, USA. ${ }^{4}$ World Vision US, Federal Way, Washington, USA. ${ }^{5}$ World Vision International, Maputo, Mozambique. ${ }^{6}$ Department of Pediatrics, Division of Infectious Diseases, Vanderbilt University, Nashville, Tennessee, USA.}

Received: 7 July 2015 Accepted: 15 December 2015 Published online: 17 December 2015

\section{References}

1. Baker P, Friel S. Processed foods and the nutrition transition: evidence from Asia. Obes Rev. 2014;15(7):564-77.

2. Popkin BM. The nutrition transition and its health implications in lower-income countries. Public Health Nutr. 1998;1(1):5-21.

3. Hawkes C. Uneven dietary development: linking the policies and processes of globalization with the nutrition transition, obesity and diet-related chronic diseases. Glob Health. 2006;2:4. 
4. Black RE, Victora CG, Walker SP, Bhutta ZA, Christian P, de Onis M, et al. Maternal and child undernutrition and overweight in low-income and middle-income countries. Lancet. 2013;382(9890):427-51.

5. Davis TP, Wetzel C, Hernandez Avilan E, de Mendoza L, Chase RP, Winch PJ, et al. Reducing child global undernutrition at scale in Sofala Province, Mozambique, using Care Group Volunteers to communicate health messages to mothers. Glob Health Sci Pract. 2013;1(1):35-51.

6. Report On The Millennium Development Goals: Republic of Mozambique. 2010. http://www.undp.org/content/dam/undp/library/MDG/english/ MDG\%20Country\%20Reports/Mozambique/mozambique_september2010.pdf. Accessed 15 Dec 2014.

7. UN Data. Prevalence of stunting (moderate and severe) - WHO. 2013. http:// data.un.org/Data.aspx?d=SOWC\&f=inID:106. Accessed 15 Dec 2014.

8. United Nations Development Programme. UNDP Open Data. https://www. mz.undp.org. Accessed 16 Dec 2015.

9. UNICEF. Child poverty and disparities in Mozambique 2010. 2011. http://www.unicef.org.mz/cpd/documents/Child-Poverty-2010.pdf. Accessed 15 Dec 2014.

10. UNICEF. Improving Child Nutrition: The Achievable Imperative for Global Progress. 2013 http://www.unicef.org/gambia/Improving_Child_Nutrition_-_ the_achievable_imperative_for_global_progress.pdf. Accessed 24 Feb 2015.

11. The World Bank. Population, total. 2015. http://data.worldbank.org/indicator/ SP.POP.TOTL?order=wbapi_data_value_2011+wbapi_data_value\&sort=desc. Accessed 12 Mar 2015.

12. Psaki S, Bhutta ZA, Ahmed T, Ahmed S, Bessong P, Islam M, et al. Household food access and child malnutrition: results from the eight-country. Popul Health Metr. 2012;10(1):24.

13. Tiwari R, Ausman LM, Agho KE. Determinants of stunting and severe stunting among under-fives: evidence from the 2011 Nepal Demographic and Health Survey. BMC Pediatr. 2014;14:239.

14. Rannan-Eliya RP, Hossain SMM, Anuranga C, Wickramasinghe R, Jayatissa R, Abeykoon ATPL. Trends and determinants of childhood stunting and underweight in Sri Lanka. Ceylon Med J. 2013;58(1):10-8.

15. McDonald CM, McLean J, Kroeun H, Talukder A, Lynd LD, Green TJ. Household food insecurity and dietary diversity as correlates of maternal and child undernutrition in rural Cambodia. Eur J Clin Nutr. 2014. doi:10.1038/ejen.2014.161.

16. Remans R, Pronyk PM, Fanzo JC, Chen J, Palm C, Nemser B, et al. Multisector intervention to accelerate reductions in child stunting: an observational study from 9 sub-Saharan African countries. Am J Clin Nutr. 2011;94(6):1632-42.

17. Korkalo L, Freese R, Fidalgo L, Selvester K, Ismael C, Mutanen M. A cross-sectional study on the diet and nutritional status of adolescent girls in Zamb?zia Province, Mozambique (the ZANE Study): design, methods, and population characteristics. JMIR Res Protoc. 2014:3(1):e12.

18. Fotso JC, Madise N, Baschieri A, Cleland J, Zulu E, Kavao Mutua M, et al. Child growth in urban deprived settings: does household poverty status matter? At which stage of child development? Health Place. 2010;18:375-84.

19. United Nations. The Millennium Development Goals Report 2014. 2014. http://mdgs.un.org/unsd/mdg/Resources/Static/Products/Progress2014/ English2014.pdf. Accessed 11 Dec 2014.

20. Horton R. Maternal and child undernutrition: an urgent opportunity. Lancet. 2008;371(9608):179.

21. World Health Organization. WHO | Moderate malnutrition. http://www.who. int/nutrition/topics/moderate_malnutrition/en/. Accessed 15 Dec 2014.

22. Vergara A, Blevins M, Vaz L, Manders EJ, Gonzalez-Calvo L, Arregui C, et al. SCIP - Ogumaniha: Improving health and livelihoods of children, women and families in the Province of Zambézia, Republic of Mozambique - Phase I and II: Zambézia-wide. 2011. http://globalhealth.vanderbilt.edu/manage/wp-content/ uploads/phase1and2 report.pdf. Accessed 3 July 2015

23. Black RE, Allen LH, Bhutta ZA, Caulfield LE, de Onis M, Ezzati M, et al. Maternal and child undernutrition: global and regional exposures and health consequences. Lancet. 2008;371(9608):243-60.

24. Swindale A, Bilinsky P. Household dietary diversity score (HDDS) for measurement of household food access: Indicator guide. Washington, D.C: Academy for Educational Development; 2005. Food and Nutrition Technical Assistance Project.

25. Mukolo A, Blevins M, Victor B, Paulin HN, Vaz LME, Sidat M, et al. Community stigma endorsement and voluntary counseling and testing behavior and attitudes among female heads of household in Zambézia Province, Mozambique. BMC Public Health. 2013:13(1):1155.

26. StataCorp. Stata statistical software: release 13. College Station, TX: StataCorp LP; 2013.

27. World Health Organization. Nutrition Landscape Information System (NLIS) country profile indicators: interpretation guide. 2010. http://www.who.int/ nutrition/nlis_interpretation_guide.pdf. Accessed 15 May 2015.

28. World Health Organization. Description - Child growth indicators and their interpretation. 2015. http://www.who.int/nutgrowthdb/about/introduction/ en/index2.html. Accessed 21 Jun 2015.

29. Bhutta ZA, Ahmed T, Black RE, Cousens S, Dewey K, Guigliani E, et al. What works? Interventions for maternal and child undernutrition and survival. Lancet. 2008;371(9610):417-40.

30. Herrador Z, Sordo L, Gadisa E, Moreno J, Nieto J, Benito A, et al. Cross-sectional study of malnutrition and associated factors among school aged children in rural and urban settings of Fogera and Libo Kemkem Districts, Ethiopia. PLoS ONE. 2014;9(9):e105880.

31. Bhutta ZA, Das JK. Interventions to address maternal and childhood undernutrition: current evidence. Nestlé Nutr Inst Workshop Ser. 2014;78:59-69.

32. Low JW, Arimond M, Osman N, Cunguara B, Zano F, Tschirley D. A food-based approach introducing orange-fleshed sweet potatoes increased vitamin A intake and serum retinol concentrations in young children in Rural Mozambique. J Nutr. 2007;137(5):1320-7.

33. Victor B, Blevins M, Green AF, Ndatimana E, Gonzalez-Calvo L, Fischer EF, et al. Multidimensional poverty in rural mozambique: a new metric for evaluating public health interventions. PLOS ONE. 2014;9(9):e108654.

34. UNICEF. Chronic Malnutrition: Stunting. nd. http://www.unicef.org/nutrition/ training/2.3/20.html. Accessed 15 May 2015

35. Simpore J, Kabore F, Zongo F, Dansou D, Bere A, Pignatelli S, et al. Nutrition rehabilitation of undernourished children utilizing Spiruline and Misola. Nutr J. 2006;5:3. doi:10.1186/1475-2891-5-3.

36. Baker KK, Dil Farzana F, Ferdous F, Ahmed S, Kumar Das S, Faruque ASG, et al. Association between moderate-to-severe diarrhea in young children in the global enteric multicenter study (GEMS) and types of handwashing materials used by caretakers in Mirzapur, Bangladesh. Am J Trop Med Hyg. 2014;91(1):181-9.

37. Assis A, Barreto M, Santos L, Fiaccone R, da Silva Gomes G. Growth faltering in childhood related to diarrhea: a longitudinal community based study. Eur J Clin Nutr. 2005;59:1317-23.

38. Ngure FM, Reid BM, Humphrey JH, Mbuya MN, Pelto G, Stoltzfus RJ. Water, sanitation, and hygiene (WASH), environmental enteropathy, nutrition, and early child development: making the links. Ann N Y Acad Sci. 2014;1308:118-28.

\section{Submit your next manuscript to BioMed Central and we will help you at every step:}

- We accept pre-submission inquiries

- Our selector tool helps you to find the most relevant journal

- We provide round the clock customer support

- Convenient online submission

- Thorough peer review

- Inclusion in PubMed and all major indexing services

- Maximum visibility for your research

Submit your manuscript at www.biomedcentral.com/submit
(O) BioMed Central 